\section{Two-Dimensional Electron Gases at Oxide Interfaces}

\section{J. Mannhart, D.H.A. Blank, H.Y. Hwang, A.J. Millis, and J.-M. Triscone}

\begin{abstract}
Two-dimensional electron gases (2DEGs) based on conventional semiconductors such as $\mathrm{Si}$ or GaAs have played a pivotal role in fundamental science and technology. The high mobilities achieved in 2DEGs enabled the discovery of the integer and fractional quantum Hall effects and are exploited in high-electron-mobility transistors. Recent work has shown that 2DEGs can also exist at oxide interfaces. These electron gases typically result from reconstruction of the complex electronic structure of the oxides, so that the electronic behavior of the interfaces can differ from the behavior of the bulk. Reports on magnetism and superconductivity in oxide 2DEGs illustrate their capability to encompass phenomena not shown by interfaces in conventional semiconductors. This article reviews the status and prospects of oxide 2DEGs.
\end{abstract}

Fundamentals: Electronic Properties of Interfaces

The realization of two-dimensional electron gases (2DEGs) in semiconductor interfaces based on Si or on III-V compounds has led to tremendous developments and successes in both understanding fundamental physics and developing new devices. As a fruit of efforts that started in the 1960s, 2DEGs with typical carrier densities ranging from $10^{10} / \mathrm{cm}^{2}$ to $10^{12} / \mathrm{cm}^{2}$ can be generated at a single heterojunction (interface between two different materials) or in doped heterostructures that form superlattices (periodic arrays of interfaces).

As shown in Figure 1 for a single heterojunction, electron gases are formed in quantum well structures with typical widths on the order of $10 \mathrm{~nm}$. The potential well perpendicular to the interface causes quantization of the electronic states. Along the plane, the carriers can move with a high mobility. Whereas these mobilities reached only $\sim 10^{4} \mathrm{~cm}^{2} /(\mathrm{V} \mathrm{s})$ in early III-V heterostructures, their top values now exceed $10^{7} \mathrm{~cm}^{2} /(\mathrm{V} \mathrm{s})$ at low temperatures. ${ }^{2}$ High mobilities and long mean free paths are essential to generate quantized Hall resistances according to the integer $^{3}$ or fractional ${ }^{4}$ quantum Hall effects (QHEs) or to use the electron gases, for example, in high-electron-mobility transistors (HEMTs).

The concept invented to attain the high mobility is to spatially separate the doping layer from the mobile carriers and thereby suppress scattering at the ionized dopants. ${ }^{5}$ The spatial separation of the conduction layer and the charge-generation layer is a principle that also offers great potential for oxide heterostructures. We note in passing that a spatial separation of dopants from conducting layers occurs in high-temperature superconductors ${ }^{6,7}$ and might be essential for their high transition temperatures. faces in oxides, including oxides with strongly correlated electrons, with atomic precision. Such interfaces can generate electron systems that nature does not produce in the bulk. The electrons interact and order at the interfaces in unique ways, so that, for example, field-effect transistors using phase transitions, novel types of quantum Hall systems, and unique superconductors can be obtained. Wellcontrolled interfaces based on oxide materials have been fabricated and are being used for a possible new generation of oxide electronic devices. They complement the interface-based bulk oxide capacitors and varistors that have been a great commercial success for many decades. The defining property of interfaces-the simple fact that they connect different materials - creates new possibilities for generating novel electronic phases.
The challenges to the materials scientists and physicists are enormous. Yet, by offering tremendous flexibility, such interfaces create emerging possibilities in designing new electronic systems. Herein, we provide an overview of a particularly interesting development that recently occurred in this field: the generation of ultrathin, or even two-dimensional, electron gases at oxide interfaces.

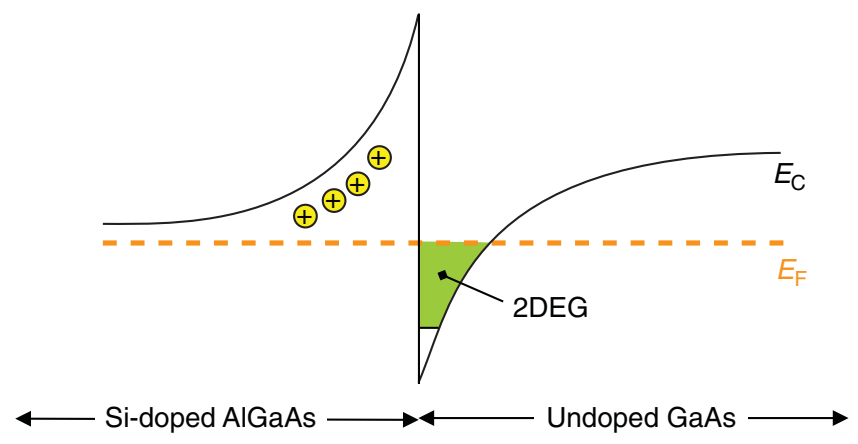

Figure 1. Band diagram showing the formation of a two-dimensional electron gas (2DEG) at a Si-doped AIGaAs-GaAs heterojunction. Note: $E_{\mathrm{F}}$ is the value of the Fermi energy, and $E_{C}$ gives the energy of the conduction band edge. 
As is well-known for interfaces involving conventional semiconductors, band bending is a phenomenon that frequently generates depletion or enhancement layers at oxide interfaces. For example, $n$-type charge carriers generated by oxygendeficient $\mathrm{SrTiO}_{3}$ can accumulate at interfaces and form sheets with high conductance. The carriers will usually be confined within a few nanometers (at $300 \mathrm{~K})$, as determined by the electricfield-dependent electrostatic screening length of the $\mathrm{SrTiO}_{3}$. An additional mechanism that can generate or remove carriers is the formation of a polar discontinuity at the interface. ${ }^{8-10}$ In bilayers consisting of a compound with charge-neutral layers and a compound with charged, polar layers, a Coulomb potential of several electronvolts can build up across the polar compound as depicted in Figure 2. If this happens, the
Coulomb energy of the polar layer can be lowered by carriers moving from the polar layer to the interface (electronic compensation). By the same token, charged defects, in particular, oxygen vacancies, can diffuse to the interface or away from it (ionic compensation). In addition to these complexities, the physics that can occur at oxide interfaces is far richer than the physics of conventional semiconductor interfaces. The richness arises from the strong correlations characteristic of oxide materials. The term "strong correlations" refers to the effects of electron-electron and electron-lattice interactions that cannot be taken into account by theories based on noninteracting electrons moving in an effective potential determined by an averaged background electronic charge. Strong correlations produce fascinating behavior in bulk materials, ranging from metal-insulator transitions to high-temperature superconductivity, and can produce an even richer range of phenomena at interfaces.

\section{Theory of Oxide Interfaces}

The theoretical study of oxide interfaces is still in its infancy. A key insight, provided by Hesper et al. ${ }^{10}$ and more recently extended by Okamoto and Millis and others,,$^{12}$ is that the presence of an interface or surface can drive an electronic reconstruction, potentially leading to novel electronic behavior not found in the bulk. The consequences of an electronic reconstruction might be useful by leading to a new superconducting or magnetic phase, for example, or they might be harmful as at interfaces in high- $T_{c}$ superconductors where a phase transition to an insulating a

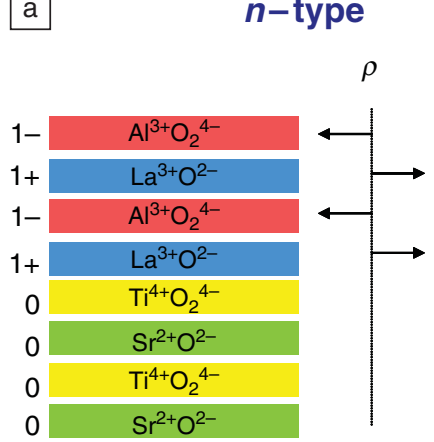

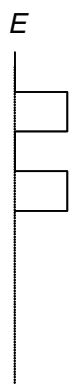

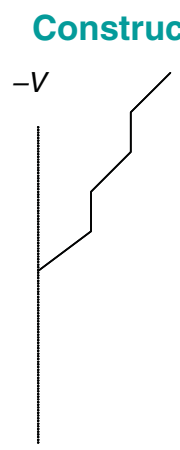

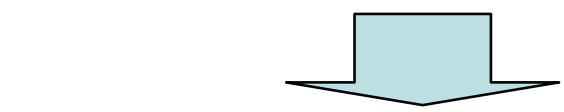

Response
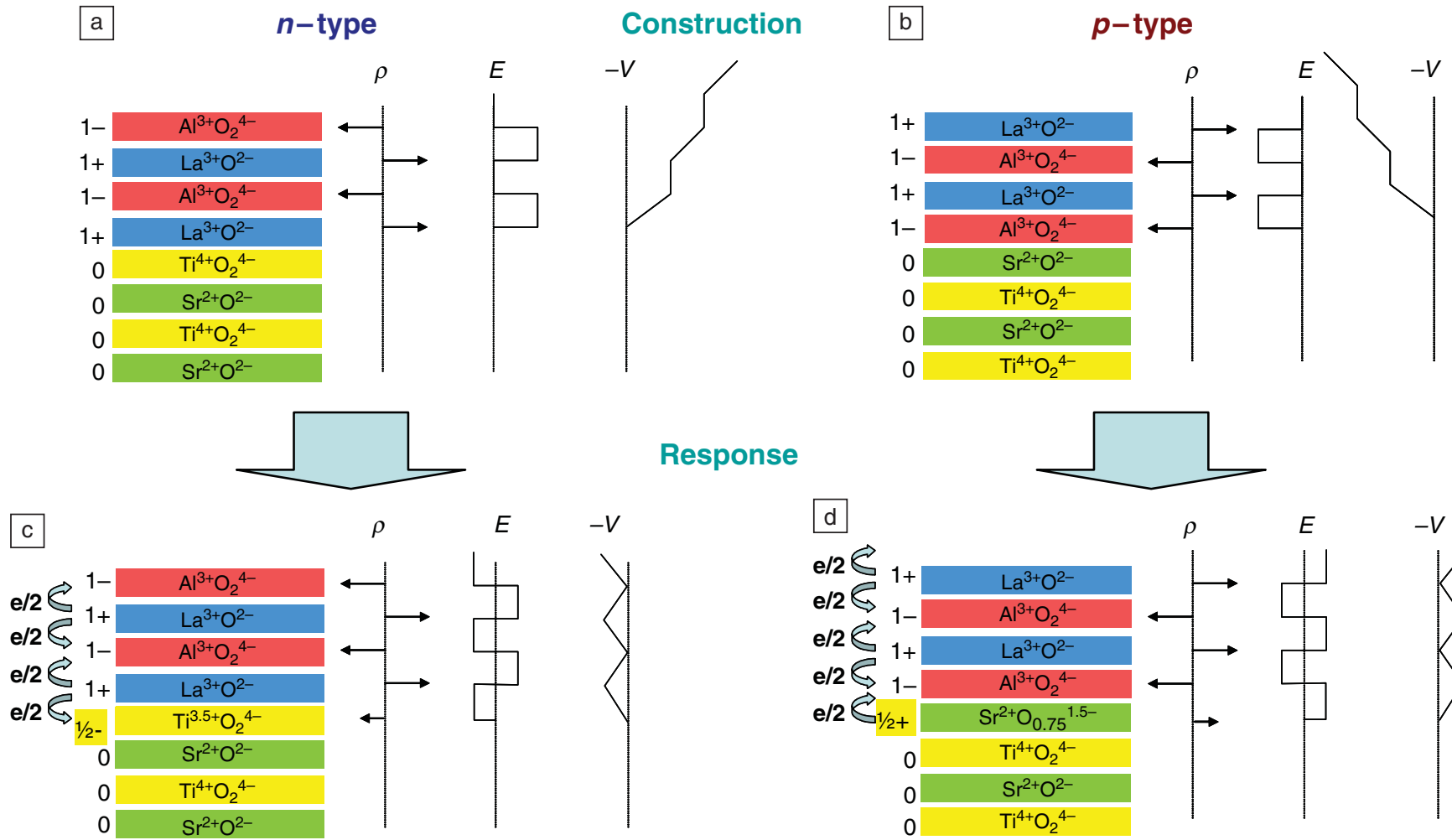

electronic reconstruction metallic interface

\section{atomic reconstruction - insulating interface}

Figure 2. Polar catastrophe illustrated for atomically abrupt (001) interfaces between $\mathrm{LaAlO}_{3}$ and $\mathrm{SrTiO}_{3}$. (a) The unreconstructed interface has neutral (001) planes in $\mathrm{SrTiO}_{3}$, but the (001) planes in $\mathrm{LaAlO}_{3}$ have alternating net charges $(\rho)$. If the interface $\mathrm{plane}$ is $\mathrm{AlO}_{2} / \mathrm{LaO} / \mathrm{TiO}{ }_{2}$, this produces a non-negative electric field $(E)$, leading in turn to an electric potential $(V)$ that diverges with thickness. (b) If the interface is instead placed at the $\mathrm{AlO}_{2} / \mathrm{SrO} / \mathrm{TiO}_{2}$ plane, the potential diverges negatively. (c) The divergence catastrophe at the $\mathrm{AlO} / 2 / \mathrm{LaO} / \mathrm{TiO}{ }_{2}$ interface can be avoided if $1 / 2$ electron per unit cell is added to the last Ti layer. This produces an interface dipole that causes the electric field to oscillate about 0 , and the potential remains finite. The upper free surface is not shown, but in this simple model, the uppermost $\mathrm{AlO}_{2}$ layer would be missing $1 / 2$ electron, which would bring the electric field and potential back to zero at the upper surface. The actual surface reconstruction is more complicated. (d) The divergence for the $\mathrm{AlO}_{2} / \mathrm{SrO} / \mathrm{TiO}_{2}$ interface can be avoided by removing $1 / 2$ electron per unit cell from the $\mathrm{SrO}$ plane in the form of oxygen vacancies. 
phase is induced, ${ }^{13-15}$ which is the key problem for the fabrication of superconducting cables. The main theoretical issues are to understand the circumstances under which a reconstruction will occur and to predict what the consequences might be. This is a challenging theoretical problem, combining fundamental issues of defect energetics and kinetics, manybody physics, and transport theory with the complications of a spatially inhomogeneous situation. Although some theoretical understanding of fundamental issues has been achieved, a comprehensive theoretical attack on the problems raised by oxide interfaces has yet to be made.

The important issues of growth, kinetics, and structure have been studied little thus far. A polarization discontinuity, as previously described, occurs at most oxide interfaces; the resulting electric field can drive the formation of a 2DEG at the interface or induce the formation of oxygen defects and interstitials. ${ }^{11,16}$ Using band-theory methods, Cen et al. ${ }^{17}$ found that an ordered array of oxygen vacancies leads to substantial changes in the lowenergy electronic structure of the $\mathrm{LaAlO}_{3} / \mathrm{SrTiO}_{3}$ interface. Empirically, the oxygen defect density is known to depend on the growth process and on the structure, but a predictive theoretical understanding of the connection is lacking. Also crucial to the question of obtaining an atomically precise interface is the understanding of the nature and energetics of defects arising from cation interdiffusion; here again, there is considerable empirical understanding, but as yet little theory. Furthermore, even in an atomically ideal interface, lattice relaxations will occur. $\mathrm{LaTiO}_{3} / \mathrm{SrTiO}_{3}$ interface calculations indicate that such relaxations are substantial ${ }^{18}$ and have a significant effect on the electronic properties. The relaxations drive an orbitally ordered phase in which the electronic density is preferentially concentrated in one of several degenerate or nearly degenerate atomic orbitals of the transition metal ion (here, Ti)..$^{19}$

Most theoretical work to date has concentrated on the electronic properties of hypothetical ideal interfaces and has involved either many-body studies of model systems such as the Hubbard model $^{20,21}$ or band-theory studies based, for example, on the local density approximation (LDA) or the LDA+ $U$ approximation. These are calculations that approximate the electronic structure in terms of the solution to an equation for noninteracting particles moving in a potential. The solution is determined selfconsistently by the total electronic charge density. In the LDA+ $U$ case, an extra inter- action expressed in terms of the Hubbard-Slater-Kanamori parameters $U$ and $J$, is included. ${ }^{17-19,22-24}$ The theoretically appealing case (occurring, for example, in $\mathrm{LaTiO}_{3} / \mathrm{SrTiO}_{3}$ ), in which the interface is defined by electrically inert counterions and the basic network that supports electrical conduction (e.g., Ti-O) is unchanged across the interface, has been most extensively studied. However, many of the insights obtained from such studies should be transferable to cases such as the $\mathrm{LaAlO}_{3} / \mathrm{SrTiO}_{3}$ interface, where one component has a large bandgap so that the 2DEG resides on only one side of the interface. The main difference would be in the values obtained by the transition-metal Ti valences: For example, in $\mathrm{LaTiO}_{3} / \mathrm{SrTiO}_{3}$, the Ti valence necessarily changes from $3+$ to $4+$ (i.e., from one to zero $d$ electrons per Ti atom), but in $\mathrm{LaAlO}_{3} / \mathrm{SrTiO}_{3}$, the effective valence of any $\mathrm{Ti}$ site may be much larger than 3.5 (i.e., much less than $1 / 2$ electron per $\mathrm{Ti}$ atom).

The most basic question concerns the electronic charge distribution. Elementary electrostatic considerations imply that the total charge is determined by the polarization discontinuity, but the confinement length in the direction transverse to the interface is an issue. Both model-system and band-theory calculations suggest that almost all of the charge is confined within about two unit cells of the interface, $, 8,19,22-26$ although, in the nearly ferroelectric material $\mathrm{SrTiO}_{3}$, a very small-amplitude, slowly decaying tail of charge extends farther from the interface but contains less than $10 \%$ of the total charge. $18,19,23$

The predicted tight confinement of charge to the near-interface region suggests that the local atomic structure at the interface is crucial to the properties of the electron gas. It is therefore not surprising that the carrier mobilities are lower at the interface than in bulk $\mathrm{SrTiO}_{3}$. Specifically, the highest reported bulk mobility in $\mathrm{SrTiO}_{3}$ is $22,000 \mathrm{~cm}^{2} /(\mathrm{V} \mathrm{s}$ ) (for electrons) at $2 \mathrm{~K} .{ }^{27}$ At room temperature, it is $6 \mathrm{~cm}^{2} /(\mathrm{V} \mathrm{s})$. For $\mathrm{LaAlO}_{3} / \mathrm{SrTiO}_{3}$ interfaces grown at oxygen pressures above $10^{-5}$ mbar, electron mobilities of $6 \mathrm{~cm}^{2} /(\mathrm{V} \mathrm{s})$ and 1,200 $\mathrm{cm}^{2} /(\mathrm{V} \mathrm{s})$ have been reported at $300 \mathrm{~K}$ and at $4.2 \mathrm{~K}$, respectively. ${ }^{28}$ The highest reported bulk mobility of GaAs is 300,000 $\mathrm{cm}^{2} /(\mathrm{V} \mathrm{s})$ (for electrons) at $50 \mathrm{~K} .{ }^{29}$ At room temperature, it is $8,500 \mathrm{~cm}^{2} /(\mathrm{V} \mathrm{s})$.

The electronic physics of many transition-metal oxides is characterized by partly filled, orbitally degenerate $d$ shells, and both orbital ordering and fluctuations play important roles in the physics. The lowered spatial symmetry near an interface might be expected to lead to addi- tional crystal field splitting; however, in the cases studied so far, these effects have been found to be negligible. ${ }^{19}$

According to the model calculations, the charge density induced at most oxide interfaces is $1 / 2$ electron per unit cell. This charge density is typically spread over several layers, corresponding to several partially filled subbands. The electronic states in these bands arise from transition $d$ levels and are expected to be strongly correlated. ${ }^{20}$ Most theoretical calculations suggest that the correlations are not strong enough to drive a metal-insulator transition in the 2DEG, although the calculations of Reference 23 predict that, in some circumstances, charge ordering occurs, leading to insulating behavior. In a metallic but strongly correlated system, the electron spectrum consists of a coherently propagating quasiparticle part, which can be characterized by its velocity, and an incoherent, essentially localized part, appearing in photoemission spectra as a nondispersive "shakeoff" band separated from the Fermi energy by 1-2 eV. Calculations suggest that the shakeoff band should be observable. ${ }^{30}$

An important question concerns the nature of the ground state of the interface 2DEG. Theoretical techniques are not yet at the point where reliable predictions can be made for unconventional superconductivity. Magnetism, orbital, and charge order can be studied. Extensive modelsystem studies ${ }^{19,31}$ as well as LDA+ $U$ calculations suggest the following general guideline as a good starting point: the electronic phase occurring in each layer can be determined by finding the layer charge density and then referring to the bulk phase diagram to determine the phase appropriate for this density. Thus, in the majority of cases, ferromagnetism is predicted $19,23,32$ because partially filled strongly correlated bands are generically predicted to be ferromagnetic. The experimental status of this prediction remains unclear.

\section{Fabrication}

Although new electronic phases formed by electronic reconstruction at oxide interfaces are already a fascinating topic from the theoretical point of view, it is especially intriguing that, in recent years, it has become possible to fabricate such interfaces with atomic precision and measure their properties. Several breakthroughs in the growth of heterostructures of complex oxides occurred in the past 10 to 20 years. In particular, the capabilities to grow complex oxide heterostructures by molecular beam epitaxy (MBE) or by pulsed laser deposition (PLD), to terminate oxide 
substrates with desired layers, and to monitor and control the growth of oxide heterostructures by using reflection highenergy electron diffraction (RHEED) make it possible to design and grow a large variety of interfaces with sub-unitcell control.

To obtain a specific surface on a perovskite $\left(\mathrm{ABO}_{3}\right)$ substrate, such as a $\mathrm{SrTiO}_{3}$ single crystal, a chemical and thermal treatment is needed. A standard (100)oriented substrate surface obtained by cutting and polishing consists of both $\mathrm{AO}$ and $\mathrm{BO}_{2}$-terminated regions separated by half-unit-cell steps. For example, $\mathrm{SrTiO}_{3}$ substrates, as delivered by vendors contain a mixture of $\mathrm{SrO}$ - and $\mathrm{TiO}_{2}$-terminated regions. To be useful for the fabrication of well-defined interfaces, the initial substrate surface must have a known termination (either by $\mathrm{AO}$ or by $\mathrm{BO}_{2}$ ). This is achieved by chemical etching in buffered $\mathrm{HF}^{33}$ It was subsequently found that etch pits can be reduced by first soaking the substrates in water to form $\mathrm{Sr}(\mathrm{OH})_{2}$ at the surface. ${ }^{34}$ After the etching, the crystals are heated in oxygen to $950^{\circ} \mathrm{C}$. Figure 3a shows an atomic force microscopy (AFM) image of a fully $\mathrm{TiO}_{2}$-terminated $\mathrm{SrTiO}_{3}$ surface obtained in this way. Chemical procedures to produce fully SrO-terminated surfaces ${ }^{37}$ are less reproducible and have not yet found broad use. Fully SrO-terminated surfaces have, however, been obtained by deposition of $\mathrm{SrO}$ monolayers on $\mathrm{TiO}_{2}$-terminated $\mathrm{SrTiO}_{3}$ substrates $^{38}$ (Figure $3 b$ ).

Perovskite films can be grown on $\mathrm{AO}-$ or $\mathrm{BO}_{2}$-terminated substrates, and the structural and electronic properties of the two possible interfaces can be readily compared. Using PLD, layer-by-layer growth of perovskites such as $\mathrm{LaAlO}_{3}$ can be achieved by ablating from a singlecrystal $\mathrm{LaAlO}_{3}$ target, for example using a $\mathrm{KrF}$ excimer laser operated at $1 \mathrm{~Hz}$ with a fluence of $\sim 1.3 \mathrm{~J} / \mathrm{cm}^{2}$. Typical deposition conditions are a substrate temperature of $T_{\mathrm{dep}} \approx 700-850^{\circ} \mathrm{C}$ and oxygen pressures of $10^{-5}-10^{-4} \mathrm{mbar}$. The film growth is usually monitored by RHEED. 39,40 The oscillations of the RHEED intensity during the initial growth of the first unit cells are shown in Figure 4 for $\mathrm{LaAlO}_{3}$ layers grown on $\mathrm{TiO}_{2}$ and $\mathrm{SrO}$-terminated $\mathrm{SrTiO}_{3} \cdot{ }^{35,36}$ For both terminations, the oscillations indicate a two-dimensional layer-by-layer growth mode. This two-dimensional layer-bylayer growth of $\mathrm{LaAlO}_{3}$ can be observed up to thicknesses of $\sim 50 \mathrm{~nm}$. After the sample has been cooled, the 3\% lattice mismatch between $\mathrm{LaAlO}_{3}$ and $\mathrm{SrTiO}_{3}$ typically causes cracking for films thicker than $~ 10-20 \mathrm{~nm}$. Figure 5 shows a scanning force microscopy image of a $\mathrm{LaAlO}_{3}$ film grown on $\mathrm{TiO}_{2}$-terminated $\mathrm{SrTiO}_{3}$ together with a corresponding scan profile. ${ }^{35,36}$ Both reveal smooth terraces with clear unit-cell steps, strongly suggesting the presence of $\mathrm{LaAlO}_{3}$ surfaces with one type of termination.
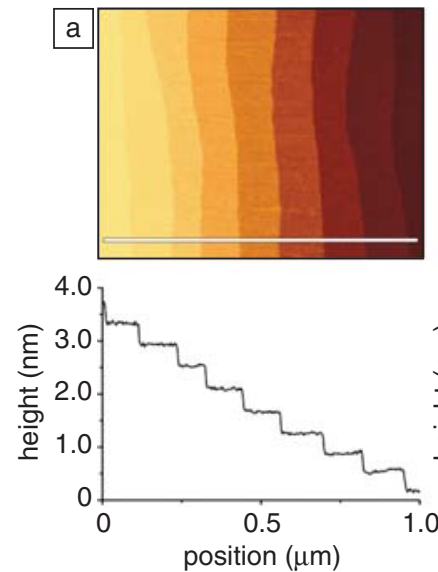

Even though the structural properties of the $\mathrm{LaAlO}_{3}$ thin films grown on the two types of terminated $\mathrm{SrTiO}_{3}$ substrates are similar as observed by RHEED and by $x$-ray diffraction, the electronic properties differ completely. The temperature depend-
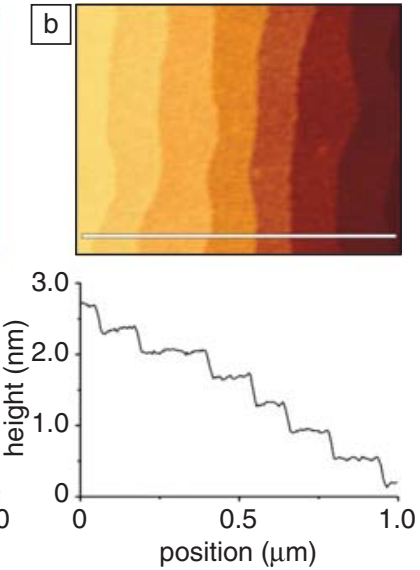

Figure 3. Surface analysis of $\mathrm{SrTiO}_{3}$ substrates by atomic force microscopy (AFM): AFM micrographs and surface roughness analysis results from (a) a chemically and thermally treated fully $\mathrm{TiO}_{2}$-terminated surface and (b) a pulsed-laser-deposited SrO-terminated surface. The figure illustrates the high quality in which $\mathrm{SrTiO}_{3}$ substrates of both termination types can be prepared.
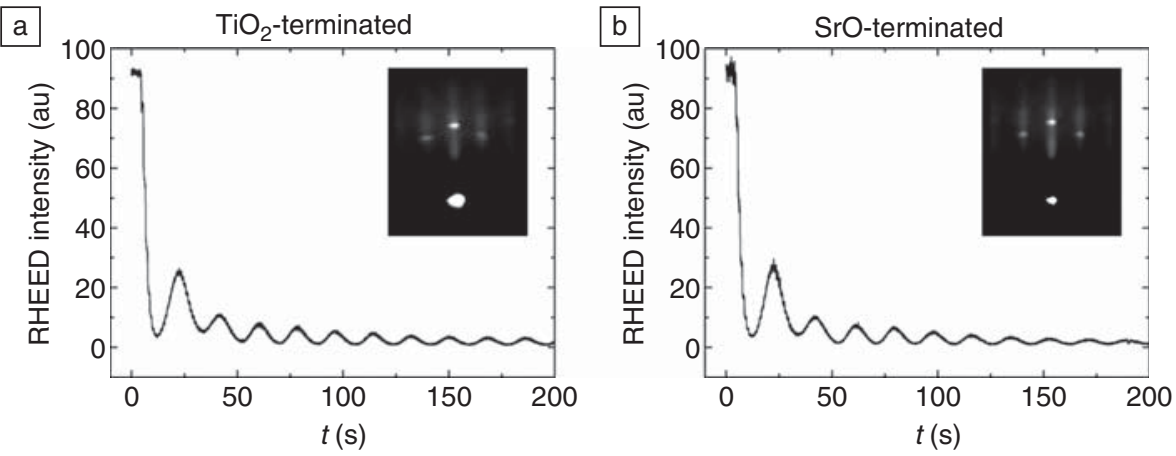

Figure 4. Reflection high-energy electron diffraction (RHEED) intensity monitoring during growth of $\mathrm{LaAlO}_{3}$ on (a) $\mathrm{TiO}_{2}$ - and (b) SrO-terminated $\mathrm{SrTiO}_{3}$ surfaces. The RHEED patterns shown in the insets were taken after the growth of 26 unit cells and reveal clear two-dimensional RHEED spots.
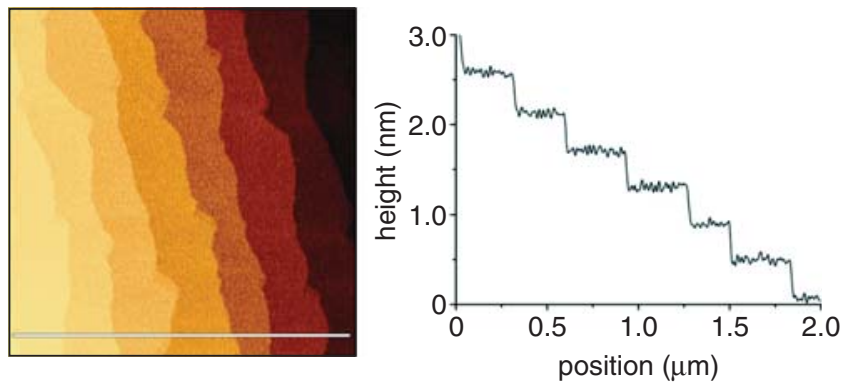

Figure 5. Scanning force microscopy image of a 26-unit-cell-thick $\mathrm{LaAlO}_{3}$ film grown on $\mathrm{TiO}_{2}$-terminated $\mathrm{SrTiO}_{3}$. The scan profile taken at the grey line (left) shows smooth terraces with unit-cell steps. 
ence of the resistances is shown in Figure 6 for both interface types. At room temperature, the resistances of the two interfaces differ by a factor of $\sim 10^{3}$. Whereas the $\mathrm{LaO} / \mathrm{TiO}_{2}$ interface shows metallic behavior, the $\mathrm{AlO}_{2} / \mathrm{SrO}$ interface is insulating. This behavior was first reported by Ohtomo and Hwang 41 and provides evidence that oxygen vacancies alone cannot be the reason for the conductivity. Only when the oxygen pressure during deposition is very low (typically a few times $10^{-6}$ mbar or smaller) is doping by oxygen vacancies in $\mathrm{SrTiO}_{3}$ the dominant carrier generation mechanism. $42-44$

The $\mathrm{LaAlO}_{3}$ layer plays a key role in generating a conducting electron gas at the $\mathrm{LaO} / \mathrm{TiO}_{2}$ interface. As revealed in Reference 45 , for $\mathrm{SrTiO}_{3} / \mathrm{LaAlO}_{3} / \mathrm{SrTiO}_{3}$ trilayers, in which the $\mathrm{LaAlO}_{3}$ layer separates a $p$-from an $n$-doped interface, the sample resistivity is a function of the $\mathrm{LaAlO}_{3}$ thickness. Below a separation distance of six unit cells, the sample resistivity is found to increase steadily with decreasing $\mathrm{LaAlO}_{3}$ thickness. Interfaces spaced by one unit cell still conduct well, but their resistance is a factor of 3-4 times higher than the resistance of samples with thick $\mathrm{LaAlO}_{3}$ layers.

Thiel et al. ${ }^{28}$ found that the resistivity of $\mathrm{LaAlO}_{3} / \mathrm{SrTiO}_{3}$ bilayers changes abruptly as a function of the $\mathrm{LaAlO}_{3}$ thickness. If the $\mathrm{LaAlO}_{3}$ layer is less than four unit cells thick, the otherwise metallic $\mathrm{LaAlO}_{3}$ / $\mathrm{SrTiO}_{3}\left(\mathrm{TiO}_{2}\right.$-terminated) interfaces are highly insulating (Figure 7). It is the presence of the fourth unit cell of the insulator

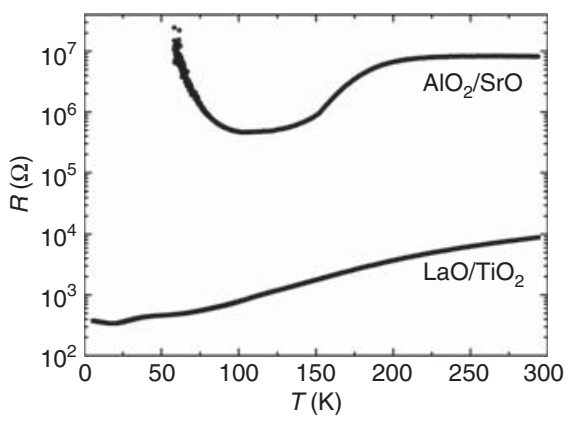

Figure 6. Temperature dependence of the resistance $(R)$ of 26-unit-cell-thick $\mathrm{LaAlO}_{3}$ films grown on (100) $\mathrm{SrTiO}_{3}$ with a $\mathrm{TiO}_{2}$-terminated surface $\left(\mathrm{LaO} / \mathrm{TiO}_{2}\right.$ interface) and a SrO-terminated surface $\left(\mathrm{AlO}_{2} / \mathrm{SrO}\right.$ interface $)$, both grown at $850^{\circ} \mathrm{C}$ and $3 \times 10^{-5} \mathrm{mbar}$ oxygen pressure. The difference in the resistances of the two samples provides evidence that the conductance is controlled by the termination of the $\mathrm{SrTiO}_{3}$ surface rather than by its oxygen content.
$\mathrm{LaAlO}_{3}$ that turns the interface into a metal. A similar behavior is shown by $n$ type $\mathrm{LaVO}_{3} / \mathrm{SrTiO}_{3}$ interfaces, in which five unit cells of $\mathrm{LaVO}_{3}$ are needed to switch the interface to become conducting. ${ }^{46}$ The existence of this critical thickness is consistent with carrier generation by the polar discontinuity.

The thickness dependence also provides the basis for a new technique to pattern the interfaces. Using photolithography or electron-beam lithography and liftoff, conducting lines are patterned into an insulating background by growing the $\mathrm{LaAlO}_{3}$ to a thickness of six unit cells in the areas to be conducting and to a thickness of two unit cells in the insulating areas ${ }^{47}$ (Figure 8). Patterning with a resolution in the nanometer range has been achieved by scanning the $\mathrm{LaAlO}_{3}$ surface with a conducting, voltage-biased AFM tip, thereby writing and erasing conducting lines. ${ }^{17}$

A central issue in understanding the atomic and electronic structure of oxide interfaces is the need to probe these features with high spatial resolution. Scanning transmission electron microscopy (STEM) coupled with electron energy-loss spectroscopy (EELS) is well-suited for this task, with atomic resolution now achievable in samples with thin cross sections. In dealing with oxides, and particularly in determining the origin of the conducting nature of the $\mathrm{LaAlO}_{3} / \mathrm{SrTiO}_{3}$ interface, an accurate measure of the oxygen stoichiometry and its electronic consequences is a vital but extremely challenging task. Although the oxygen lattice has recently been directly

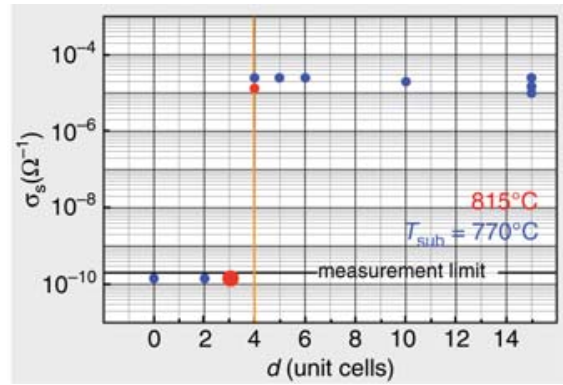

Figure 7. Influence of the $\mathrm{LaAlO}_{3}$ thickness on the electronic properties of the $\mathrm{LaAlO}_{3} / \mathrm{SrTiO}_{3}$ interface, showing an increase in conductivity when the thickness reaches four unit cells. The sheet conductances $\left(\sigma_{\mathrm{s}}, 300 \mathrm{~K}\right)$ of the heterostructures are plotted as a function of the thickness of the $\mathrm{LaAlO}_{3}$ layers in unit cells. The data shown in blue and red are those of samples grown at substrate temperatures $\left(T_{\text {sub }}\right)$ of $770^{\circ} \mathrm{C}$ and $815^{\circ} \mathrm{C}$, respectively. imaged using spherical-aberrationcorrected TEM, the signal intensity is far too weak to detect small oxygen vacancy levels. ${ }^{48}$ Therefore, oxygen vacancies have instead been probed indirectly using EELS. ${ }^{49}$

In $\mathrm{SrTiO}_{3}$, the $\mathrm{O} \mathrm{K}$-edge fine structure has been used to probe oxygen vacancies with a resolution limit of $\sim 4 \%$; the Ti $L$-edge can detect the electrons donated by oxygen vacancies down to an effective resolution of $\sim 1 \%$. This resolution is likely a practical lower bound to these techniques, as it corresponds to just a few oxygen vacancies across the entire sample, the thickness of which is limited by the need to prepare electron-transparent cross sections.

Using this approach, the $\mathrm{LaAlO}_{3} / \mathrm{SrTiO}_{3}$ interface has been examined in detail. ${ }^{11}$ For the conducting $n$-type interface, a significant $\mathrm{Ti}^{3+}$ component was measured at the interface, with very few oxygen vacancies. By contrast, at the insulating $p$-type interface, little $\mathrm{Ti}^{3+}$ was observed despite a large number of oxygen vacancies. These results are consistent with electronic reconstructions driven to resolve the polar discontinuity of the interface. It should be noted, however, that oxygen vacancy levels below the $\sim 1 \%$ detection threshold can still contribute to a significant conductivity in bulk $\mathrm{SrTiO}_{3} \cdot 50$

\section{Systems Investigated}

With the technical advances in oxide film growth described in the previous section, a number of interesting oxide heterointerfaces have recently been developed. One highlight is the observation of the integer quantum Hall effect in the high-mobility electron gas at the $\mathrm{ZnO} /(\mathrm{Mg}, \mathrm{Zn}) \mathrm{O}$ heterointerface. (See the article by Kamiya and Kawasaki in this

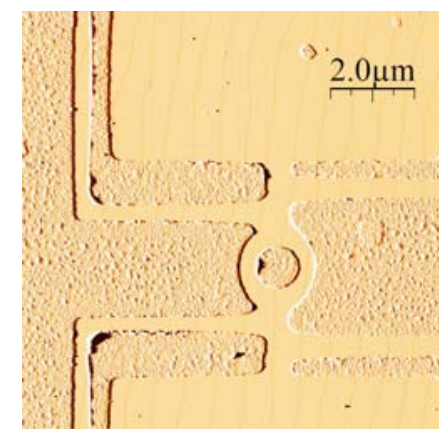

Figure 8. Atomic force microscopy image of a ring defining a quasi-twodimensional electron gas (quasi2DEG) at a 2DEG with a diameter of $1.2 \mu \mathrm{m}$ and a track width of $220 \mathrm{~nm}$ at a $\mathrm{LaAlO}_{3} / \mathrm{SrTiO}_{3}$ interface. Each half of the ring comprises one $\mathrm{LaAlO}_{3}$ terrace. 
issue.) These conducting states are derived from $s p$ hybridization, as in other nonoxide compound semiconductors such as GaAs. Here, we focus on systems that exhibit the $d$-orbital character of transition-metal oxides.

The $\mathrm{LaAlO}_{3} / \mathrm{SrTiO}_{3}$ interface is arguably the most explored interface with a conducting electron gas. The reason that numerous studies focus on this system is the ease with which robust, highly insulating films of the large bandgap insulator can be grown and the fact that the interface shows the intriguing properties described in this article. In these studies, several important questions have been addressed, among them, what is the nature of the conduction mechanism? Because some reports ${ }^{44}$ point to bulk three-dimensional conduction or thick oxygen-depleted layers, ${ }^{43}$ a second question is, what is the thickness of the conducting layer? Another key issue is the nature of the ground state of the interface electronic system.

As reported in Reference 51, signatures of a ferromagnetic ground state have been found in the magnetoresistance behavior of $\mathrm{LaAlO}_{3} / \mathrm{SrTiO}_{3}$ samples grown at very high oxygen pressures. In a series of samples prepared in Augsburg, Germany, and Geneva, Switzerland, however, lowtemperature measurements revealed a superconducting condensate to be the ground state of the samples investigated. ${ }^{52}$ The measured critical temperature equals 200 mK (Figure 9).

This discovery allows some of the issues raised to be answered. Critical field measurements with magnetic fields aligned parallel and perpendicular to the interface plane reveal a large anisotropy. The in-plane coherence length is estimated to be 50-100 nm and the superconducting sheet to be only a few nanometers thick (Figure 10). Because the superconducting thickness is much less than the in-plane coherence length, the interface is expected to behave as a two-dimensional superconductor such as described by the BerezinskiiKosterlitz-Thouless theory. Indeed, current-voltage characteristics are consistent with two-dimensional behavior. ${ }^{52}$ In fieldeffect transistor configurations, ${ }^{53-55}$ perpendicular electric fields allow the sheet carrier density of $\mathrm{LaAlO}_{3} / \mathrm{SrTiO}_{3}$ interfaces to be substantially modulated in both the normal ${ }^{28}$ and superconducting 56 states. At low temperatures, an insulatorto-superconductor phase transition can be induced with electric fields, thereby enabling the phase diagram to be mapped out. 56
One possibility discussed to reconcile the findings of ferromagnetic and superconducting ground states is the possible dependence of the ground state on the doping level. Measurements in the electric-field-induced insulating state did not, however, reveal hysteresis in the magnetoresistance measurements. Finally, it might well be that these fascinating systems reveal, in their normal and superconducting states, signatures of inversion symmetry breaking at the interface. Experiments to address this question are in progress.

The $\mathrm{LaTiO}_{3} / \mathrm{SrTiO}_{3}$ interface already discussed (Figure 11) provides an interesting contrast to the $\mathrm{LaAlO}_{3} / \mathrm{SrTiO}_{3}$ interface. Rather than an interface between two band insulators, $\mathrm{LaTiO}_{3}$ is a Mott insulator in which strong electron correlations open a gap despite the high carrier density of one electron per site. Furthermore, because the Ti lattice is common to both constituents, only one type of chemical interface exists. Atomically abrupt superlattices and interfaces between $\mathrm{LaTiO}_{3}$ and $\mathrm{SrTiO}_{3}$ are metallic, and the charge distribution has been measured to extend significantly beyond the chemical interface (Figure 11).57 This charge distribution reflects a minimization of the free energy of the electrons and has a number of unusual contributions. Theoretical models show a general tendency toward orbital and spin ordering with increasing interaction strength, 32,58 as well as a significant

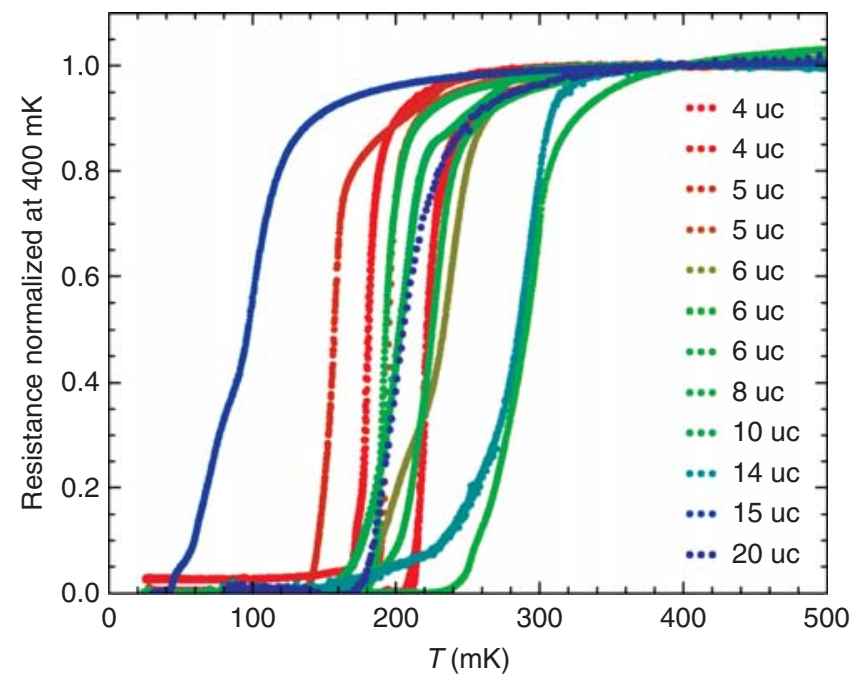

Figure 9. Transport measurements of the resistances of several $\mathrm{LaAlO}_{3} / \mathrm{SrTiO}_{3}$ heterostructures with a variety of $\mathrm{LaAlO}_{3}$ thicknesses measured in unit cells (uc). The resistances are normalized to the values measured at $400 \mathrm{mK}$. The figure shows that, in such samples, a superconducting transition with a critical temperature of $\sim 200 \mathrm{mK}$ is commonly observed.

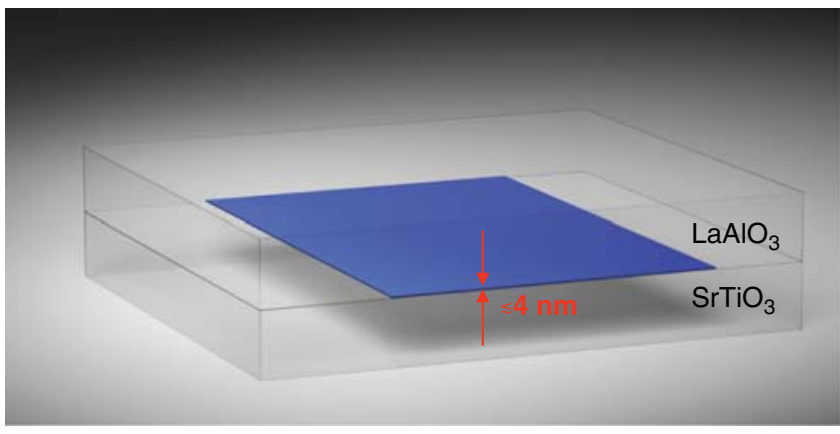

Figure 10. Sketch illustrating the generation of an ultrathin two-dimensional electron gas superconducting sheet at the $\mathrm{LaAlO}_{3} / \mathrm{SrTiO}_{3}$ interface. Based on the superconducting transition temperature and the carrier density, the thickness of the superconducting sheet is estimated to be less than $\sim 4 \mathrm{~nm}$. 


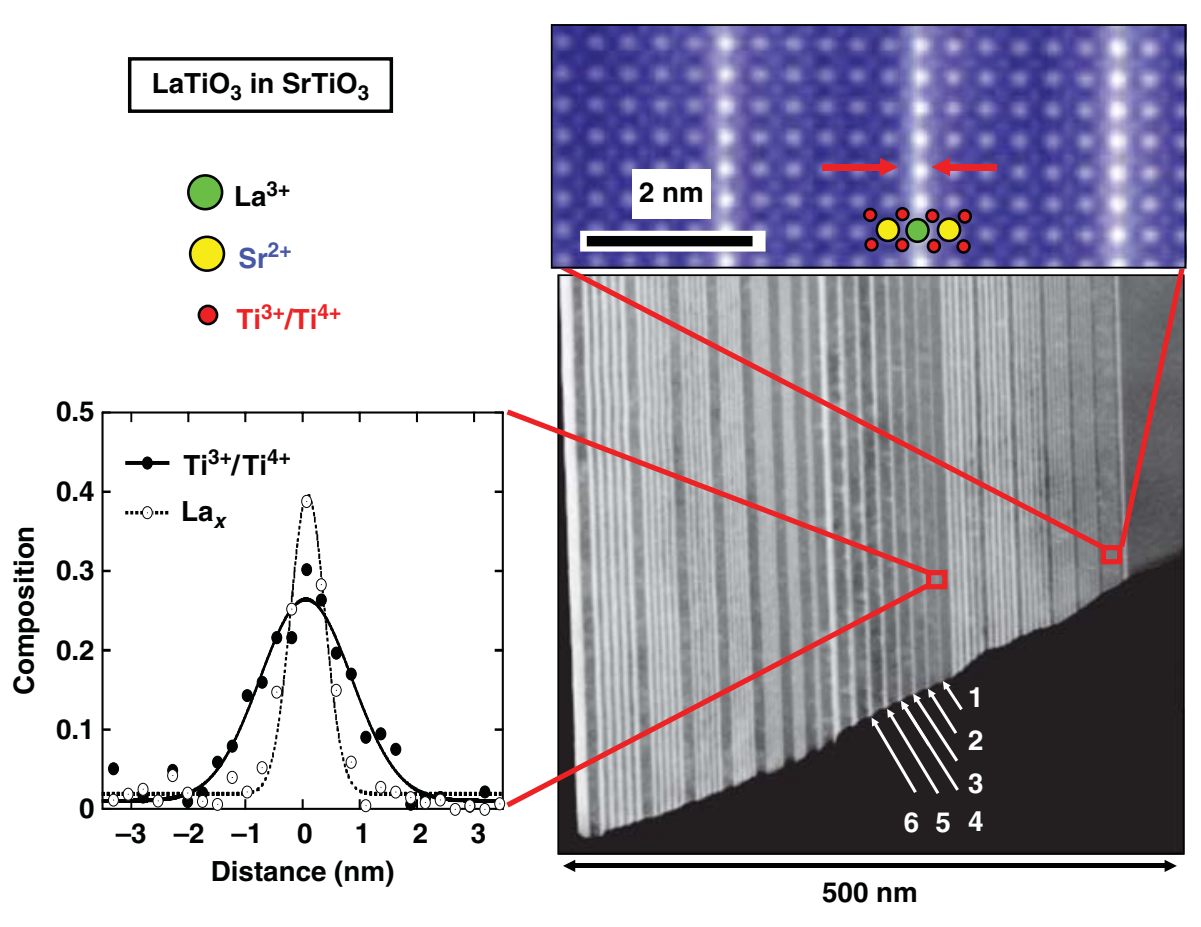

Figure 11. (Bottom right) High-angle annular dark-field scanning transmission electron microscopy image of $\mathrm{LaTiO}_{3}$ layers (bright areas) of varying thickness spaced by $\mathrm{SrTiO}_{3}$ layers, viewed down the [100] zone axis of the $\mathrm{SrTiO}_{3}$ substrate. (Top right) Enlarged view of a section of the image, with repeats of one unit cell of $\mathrm{LaTiO}_{3}$ and five unit cells of $\mathrm{SrTiO}_{3}$. (Bottom left) The La Medge is recorded simultaneously with the Ti $L$ edge, yet the $\mathrm{Ti}^{3+}$ signal is considerably wider than the La signal.

influence from a large screening polarization induced in the lattice. ${ }^{18,19}$ These characteristically unique features arise from the partially occupied $d$ orbitals at these interfaces and are qualitatively new degrees of freedom as compared to those of conventional semiconductor and metal interfaces.

An especially interesting case of interface conductivity and magnetism occurs between $\mathrm{LaMnO}_{3}$ and $\mathrm{SrMnO}_{3} .{ }^{59}$ Individually, the constituents are antiferromagnetic insulators, but at their interface, double-exchange ferromagnetism arises in analogy to the behavior of their bulk solid solution, the famous "colossal magnetoresistance" manganites. ${ }^{30}$ Here, the interface charge reconstruction was recently observed by resonant x-ray scattering, ${ }^{60}$ providing another example of novel two-dimensional states that can be induced by engineering oxide heterostructures.

\section{Conclusions and Outlook}

To assess the potential and future of 2DEGs in oxide heterostructures, it is helpful to put them into perspective by comparing them with 2DEGs in semiconductors. A precondition to the success of
2DEGs in semiconductors is their superb mobility. The reported mobilities in oxides are high at low temperatures but are orders of magnitude smaller than those in III-V heterostructures. Further, for $\mathrm{SrTiO}_{3}$ interfaces, the highest reported mobilities of $10^{4} \mathrm{~cm}^{2} /(\mathrm{Vs})(4.2 \mathrm{~K})^{41}$ seem to be associated with the presence of the highly mobile, oxygen-vacancy-doped bulk $\mathrm{SrTiO}_{3}$. Yet, in $\mathrm{ZnO}$ oxide interfaces, the mobilities are already large enough for the QHE to occur. In oxides, the work to increase the mobilities is just starting, and it will be exciting to watch whether the mobilities can be significantly enhanced. The effective masses and the sheet carrier densities are other key parameters different in semiconductor 2DEGs and electron gases at oxide interfaces. In the oxides, both are usually much larger. The electron effective mass in $\mathrm{SrTiO}_{3}$, for example, is $\sim 100$ times larger than that in GaAs. Whereas carrier densities of $10^{10}-10^{12} / \mathrm{cm}^{2}$ are typical for the semiconductors, carrier densities of $10^{13}-10^{14} / \mathrm{cm}^{2}$ are standard for oxidized $\mathrm{LaAlO}_{3} / \mathrm{SrTiO}_{3}$ interfaces. The magnetic fields needed to reach the $\mathrm{QHE}$ steps are correspondingly higher. Yet, for the $\mathrm{ZnO}$ interfaces, densities of $7 \times 10^{11}-3.7$ $\times 10^{12} / \mathrm{cm}^{2}$ have been reported. ${ }^{61} \mathrm{We}$ therefore expect that, in the future, oxide interfaces with a very large range of carrier densities will be available.

Many open questions remain. The twodimensional nature of the electron gases, for example, has been demonstrated in the normal state for only the $\mathrm{ZnO}$ system and in the superconducting state only for the $\mathrm{LaAlO}_{3} / \mathrm{SrTiO}_{3}$ interface. It would be helpful if more oxide 2DEG systems were found. To date, all analogues of the $\mathrm{LaAlO}_{3} / \mathrm{SrTiO}_{3}$ system with its conducting electron gas involve $\mathrm{SrTiO}_{3}$. Why this is the case is unclear. The 2DEGs typically have a carrier density that is significantly smaller than the predicted $1 / 2$ electron per unit cell. Why? What is the mechanism for the superconductivity of the $\mathrm{LaAlO}_{3} / \mathrm{SrTiO}_{3}$ interface? Is it the standard $\mathrm{SrTiO}_{3}$ superconductivity in a surface sheet, or is it pairing in the 2DEG induced by the $\mathrm{SrTiO}_{3}$ ? Does the superconductivity reflect the spin-orbit coupling of the $\mathrm{SrTiO}_{3}$ ? Furthermore, why has it not yet been possible to demonstrate multilayers with several 2DEGs?

Despite these open questions, the systems already available offer great opportunities. The electric field tunability allows phase diagrams to be mapped; electronic devices to be tuned; and maybe even devices, such as Josephson junctions or SQUIDs (superconducting quantum interference devices), to be fabricated. The metal-insulator transition is ready to be used for device applications. The electron gases form readily accessible model systems for two-dimensional behavior and for investigations of the Berezinskii-Kosterlitz-Thouless transition. It will be interesting to tune oxide 2DEGs by adding strong electronic correlations. The possibility of spatially separating the correlations and the conducting electron system provides a new degree of freedom for generating novel electronic phase by tuning the 2DEGs with correlation effects. Superconductors with very high transition temperatures are just one example that has been proposed. ${ }^{62,63}$ These heterostructures offer the possibility of using strong correlations occurring in oxides to generate new electronic phases at interfaces and new electronic systems with properties that cannot be achieved in the bulk. The spectrum of properties generated is enormous, and we can barely imagine the electronic effects that can and will be generated at such interfaces.

\section{Acknowledgments}

The authors gratefully acknowledge helpful discussions with A. Brinkman, D.R. Hamann, G. Hammerl, H. Hilgenkamp, 
Y. Hotta, M. Huijben, J.R. Kirtley, T. Kopp, J. Levy, D.A. Muller, N. Nakagawa, A. Ohtomo, R. Ramesh, G. Rijnders, D.G. Schlom, C.W. Schneider, T. Susaki, and S. Thiel. This work was supported by the EC (Nanoxide), the DFG (SFB 484), the ESF (THIOX), the Japan Science and Technology Agency, a Grant-in-Aid for Scientific Research on Priority Areas, the Mitsubishi Foundation, the Swiss National Science Foundation through the "National Center of Competence in Research Materials with Novel Electronic Properties-MaNEP" and Division II, and the Basic Energy Sciences division of the U.S. DOE through Grant ER-46169.

\section{References}

1. J.W. Orton, The Story of Semiconductors (Oxford University Press, Oxford, U.K., 2004).

2. L. Pfeiffer, K.W. West, H.L. Stormer, K.W.

Baldwin, Appl. Phys. Lett. 55, 1888 (1989).

3. K. von Klitzing, Rev. Mod. Phys. 58, 519 (1986).

4. H.L. Stormer, Rev. Mod. Phys. 71, 875 (1999).

5. R. Dingle, H.L. Störmer, A.C. Gossard W. Wiegmann, Appl. Phys. Lett. 33, 665 (1978).

6. Y. Tokura, T. Arima, Jpn. J. Appl. Phys. 29, 2388 (1990).

7. R.J. Cava, Science 247, 656 (1990).

8. G.A. Baraff, J.A. Appelbaum, D.R. Hamann, Phys. Rev. Lett. 38, 237 (1977).

9. W.A. Harrison, E.A. Kraut, J.R. Waldrop, R.W. Grant, Phys. Rev. B 18, 4402 (1978).

10. R. Hesper, L.H. Tjeng, A. Heeres, G.A. Sawatzky, Phys. Rev. B. 62, 16046 (2000).

11. N. Nakagawa, H.Y. Hwang, D.A. Muller, Nat. Mater. 5, 204 (2006).

12. S. Okamoto, A.J. Millis, Phys. Rev. B 70, 214531 (2004)

13. J. Mannhart, H. Hilgenkamp, Supercond. Sci. Technol. 10, 880 (1997).

14. H. Hilgenkamp, J. Mannhart, Rev. Mod Phys. 74, 485 (2002)

15. J. Mannhart, in Thin Films and Heterostructures for Oxide Electronics, S. Ogale, Ed. (Springer, New York, 2005), pp. 251-278

16. H.Y. Hwang, MRS Bull. 31, 28 (2006).

17. C. Cen, S. Thiel, G. Hammerl, C.W. Schneider, K.E. Andersen, C.S. Hellberg, J. Mannhart, J. Levy, Nat. Mater. 7, 298 (2008)

18. D.R. Hamann, D.A. Muller, H.Y. Hwang, Phys. Rev. B 73, 195403 (2006).

19. S. Okamoto, A.J. Millis, N.A. Spaldin, Phys Rev. Lett. 97, 056802 (2006).

20. S. Okamoto, A.J. Millis, Phys. Rev. B70 075101/1-12 (2004).

21. J.K. Freericks, Transport in Multilayered Nanostructures: The Dynamical Mean-Field Theory
Approach (Imperial College Press, London, 2006).

22. Z.S. Popovic, S. Satpathy, Phys. Rev. Lett. 94, 176805 (2005)

23. R. Pentcheva, W.E. Pickett, Phys. Rev. Lett. 99, 016802 (2007)

24. M.S. Park, S.H. Rhim, A.J. Freeman, Phys. Rev. B 74, 205416 (2006).

25. S. Gemming, G. Seifert, Acta Mater. 54, 4299 (2006).

26. U. Schwingenschlögl, C. Schuster, Eur. Phys. Lett. 81, 17007 (2008).

27. O.N. Tufte, P.W. Chapman, Phys. Rev. 155, 796 (1967).

28. S. Thiel, G. Hammerl, A. Schmehl, C.W. Schneider, J. Mannhart, Science 313, 1942 (2006). 29. C.M. Wolfe, G.E. Stillman, W.T. Lindley, J. Appl. Phys. 41, 3088 (1970).

30. M. Takizawa, H. Wadati, K. Tanaka, M. Hashimoto, T. Yoshida, A. Fujimori, A. Chikamatsu, H. Kumigashira, M. Oshima, K. Shibuya, T. Mihara, T. Ohnishi, M. Lippmaa, M. Kawasaki, H. Koinuma, S. Okamoto, A.J. Millis, Phys. Rev. Lett. 97, 057601 (2006).

31. C. Lin, S. Okamoto, A.J. Millis, Phys. Rev. B 73, 041104 (2006)

32. R. Pentcheva, W.E. Pickett, Phys. Rev. B 74, 035112 (2006)

33. M. Kawasaki, K. Takahashi, T. Maeda, R.T.M. Shinohara, O. Ishiyama, T. Yonezawa, M. Yoshimoto, H. Koinuma, Science 266, 1540 (1994).

34. G. Koster, B.L. Kropman, G.J.H.M. Rijnders, D.H.A. Blank, H. Rogalla, Appl. Phys. Lett. 73, 2920 (1998).

35. M. Huijben, $\mathrm{PhD}$ thesis, University of Twente, Enschede, The Netherlands (2006).

36. M. Huijben, A. Brinkman, G. Koster, G. Rijnders, H. Hilgenkamp, D.H.A. Blank, Adv. Mater., in press (available at http:/ /arxiv. org/abs/0809.1068).

37. A.G. Schrott, J.A. Misewich, M. Copel, D.W. Abraham, Y. Zhang, Appl. Phys. Lett. 79, 1786 (2001)

38. G. Koster, G. Rijnders, D.H.A. Blank, H. Rogalla, Physica C 339, 215 (2000).

39. T. Frey, C.C. Chi, C.C. Tsuei, T. Shaw, F. Bozso, Phys. Rev. B 49, 3483 (1994).

40. G.J.H.M. Rijnders, G. Koster, D.H.A. Blank, H. Rogalla, Appl. Phys. Lett. 79, 1888 (1997)

41. A. Ohtomo, H.Y. Hwang, Nature 427, 423 (2004).

42. A.S. Kalabukhov, R. Gunnarsson, J. Börjesson, E. Olsson, T. Claeson, D. Winkler, Phys. Rev. B 75, 121404 (2007)

43. W. Siemons, G. Koster, H. Yamamoto, W.A. Harrison, G. Lucovsky, T.H. Geballe, D.H.A. Blank, M.R. Beasley, Phys. Rev. Lett. 98, 196802 (2007).

44. G. Herranz, M. Basletic, M. Bibes, C. Carrétéro, E. Tafra, E. Jacquet, K. Bouzehouane,
C. Deranlot, A. Hamzic, J.-M. Broto, A. Barthélémy, A. Fert, Phys. Rev. Lett. 98, 216803 (2007).

45. M. Huijben, G. Rijnders, D.H.A. Blank, S. Bals, S. Van Aert, J. Verbeeck, G. Van Tendeloo, A. Brinkman, H. Hilgenkamp, Nat. Mater. 5, 556 (2006).

46. Y. Hotta, T. Susaki, H.Y. Hwang, Phys. Rev Lett. 99, 236805 (2007).

47. C.W. Schneider, S.Thiel, G. Hammerl, C. Richter, J. Mannhart, Appl. Phys. Lett. 89, 122101 (2006).

48. C.L. Jia, M. Lentzen, K. Urban, Science 299 870 (2003).

49. D.A. Muller, N. Nakagawa, A. Ohtomo, J.L. Grazul, H.Y. Hwang, Nature 430, 657 (2004). 50. H.P.R. Frederikse, W.R. Thurber, W.R. Hosler, Phys. Rev. 134, A442 (1964).

51. A. Brinkmann, M. Huijben, M. van Zalk, J. Huijben, U. Zeitler, J.C. Maan, W.G. van der Wiel, G. Rijnders, D.H.A. Blank, H. Hilgenkamp, Nat. Mater. 6, 493 (2007).

52. N. Reyren, S. Thiel, A. Caviglia, L. FittingKourkoutis, G. Hammerl, C. Richter, C.W. Schneider, T. Kopp, A.-S. Rüetschi, D. Jaccard, M. Gabay, D.A. Muller, J.-M. Triscone, J. Mannhart, Science 317, 1196 (2007).

53. J. Mannhart, J.G. Bednorz, K.A. Müller, D.G. Schlom, Z. Phys. B 83, 307 (1991).

54. C.H. Ahn, J.-M. Triscone, J. Mannhart, Nature 424, 1015 (2003).

55. C.H. Ahn, A. Bhattacharya, M. Di Ventra, J.N. Eckstein, C.D. Frisbie, M.E. Gershenson, A.M. Goldman, I.H. Inoue, J. Mannhart, A. Millis, A. Morpurgo, D. Natelson, J.-M. Triscone, Rev. Mod. Phys., 78, 1185 (2006).

56. A. Caviglia, N. Reyren, S. Gariglio, D. Jaccard, T. Giamarchi, J.-M. Triscone, T. Schneider, L. Benfatto, S. Thiel, G. Hammerl, J. Mannhart, "Electric Field Control of the $\mathrm{LaAlO}_{3} / \mathrm{SrTiO}_{3}$ Interface Ground State, manuscript submitted.

57. A. Ohtomo, D.A. Muller, J.L. Grazul, H.Y. Hwang, Nature 419, 378 (2002).

58. S. Okamoto, A.J. Millis, Nature 428, 630 (2004).

59. P.A. Salvador, A.-M. Haghiri-Gosnet, B. Mercey, M. Hervieu, B. Raveau, Appl. Phys. Lett. 75, 2638 (1999)

60. S. Smadici, P. Abbamonte, A. Bhattacharya, X. Zhai, B. Jiang, A. Rusydi, J.N. Eckstein, S.D. Bader, J.-M. Zuo, Phys. Rev. Lett. 99, 196404 (2007).

61. A. Tsukazaki, A. Ohtomo, T. Kita, Y. Ohno, H. Ohno, M. Kawasaki, Science 315, 1388 (2007) 62. V. Koerting, Q. Yuan, P.J. Hirschfeld, T. Kopp, J. Mannhart, Phys. Rev. B 71, 104510 (2005).

63. J. Chaloupka, G. Khaliullin, Phys. Rev. Lett 100, 016404 (2008)

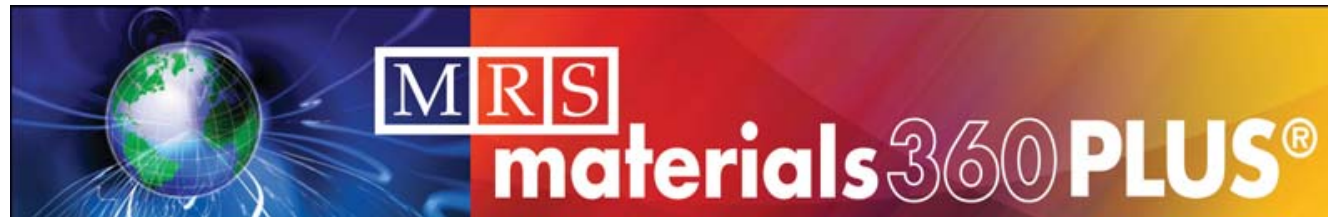

www.mrs.org/360plus
Materials News Materials Information Resources and Links Meetings Calendar and Much More! 